\title{
INTERNATIONAL MULTIWAVELENGTH CAMPAIGNS ON SHORT-TERM VARIABILITY OF OB STARS: OPTICAL POLARIMETRY
}

\author{
D. MCDAVID* \\ Limber Observatory, P.O. Box 63599, Pipe Creek TX 78063-3599
}

From 1986 through 1992, wide-band optical ( $B$ or $V$ filter) linear polarization measurements of eight Be stars and seven $O$ stars were obtained simultaneously with ultraviolet observations from $I U E$ and worldwide ground-based optical spectroscopy and photometry in a series of campaigns designed to study the short-term variability of these objects. Each campaign consisted of intensive monitoring of a few carefully chosen stars over a period of several days and nights, with the greatest possible continuity subject to the limitations of instrument scheduling, weather, and the longitudes of the observing sites.

With a typical instrumental uncertainty of about $0.03 \%$ for a single observation, no polarization variability was detected at the $3 \sigma$ level for any of the program stars. Normalized Stokes parameter plots are shown in Fig. 1, where the data points are filled circles, the mean is a cross drawn to the size of the average instrumental uncertainty of a single observation, the standard deviation is represented by a dotted ellipse centered on the mean, and three times the average instrumental uncertainty of a single observation is represented by a solid ellipse centered on the mean.

Since most of the stars showed definite signs of activity in their winds and photospheres during the time intervals covered, it appears that associated changes in polarization are uncommon, or at least too small to measure by current techniques. In some cases, weak periodicities may be present in the polarization at frequencies which match those found in the simultaneous photometric and spectroscopic data sets from the campaigns, but their significance has not yet been thoroughly evaluated.

* Guest Observer, McDonald Observatory, University of Texas at Austin; Visiting Astronomer, Cerro Tololo Inter-American Observatory, National Optical Astronomy Observatories, operated by the Association of Universities for Research in Astronomy, Inc., under contract with the National Science Foundation. 

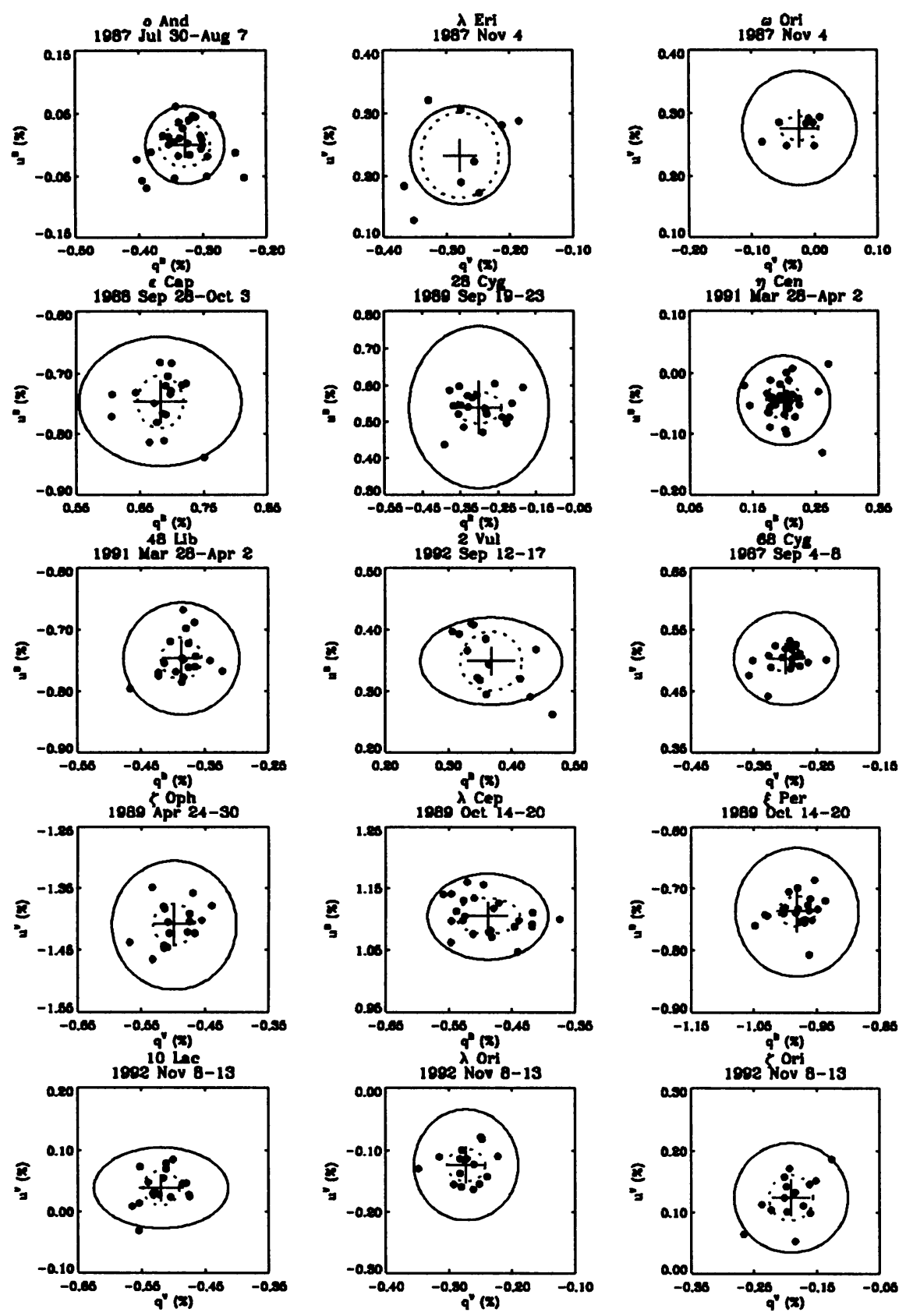

Fig. 1. Normalized Stokes parameter plots for the program stars. (The symbols are explained in the text.) 